November 1991

\title{
MULTIPLE CROSSOVER PHENOMENA AND SCALE HOPPING IN TWO DIMENSIONS
}

\author{
Michael Lässig f \\ Institut für Festkörperforschung \\ Forschungszentum Jülich \\ 5170 Jülich, Germany
}

\begin{abstract}
We study the renormalization group for nearly marginal perturbations of a minimal conformal field theory $M_{p}$ with $p \gg 1$. To leading order in perturbation theory, we find a unique one-parameter family of "hopping trajectories" that is characterized by a staircase-like renormalization group flow of the $C$-function and the anomalous dimensions and that is related to a recently solved factorizable scattering theory [1]. We argue that this system is described by interactions of the form $t \phi_{(1,3)}-\bar{t} \phi_{(3,1)}$. As a function of the relevant parameter $t$, it undergoes a phase transition with new critical exponents simultaneously governed by all fixed points $M_{p}, M_{p-1}, \ldots, M_{3}$. Integrable lattice models represent different phases of the same integrable system that are distinguished by the sign of the irrelevant parameter $\bar{t}$.
\end{abstract}

\footnotetext{
*Electronic mail: iff299@DJUKFA11, lassig@iff011.dnet.kfa-juelich.de
} 


\section{Introduction}

The simplest scale-invariant field theories in two dimensions are the series of minimal models $M_{p}(p=3,4, \ldots)$ [2], which describe the universal $(p-1)$-critical behavior of Landau-Ginzburg theories with a single bosonic field and polynomial interactions [3]. It is a difficult and widely open problem to reveal the embedding renormalization group $(\mathrm{RG})$ scenario of these fixed points, which determines the universal behavior off criticality as well as crossover phenomena. An important aspect of this problem is that in two dimensions the theory has an infinite number of integrals of motion not only at the RG fixed points, where it is conformally invariant [2], but on a larger submanifold of theory space. The precise extent of this manifold of integrability is unknown, but it does contain some perturbations of a critical point $M_{p}$ by a single scaling field [4].

If such a perturbation is relevant, it will either induce a crossover to another critical point of lower criticality or lead to purely massive infrared behavior. In the latter case, the exact factorizable $S$-matrix can be conjectured [4], which in principle determines all scaling functions associated to that RG trajectory. At least some properties of this scaling regime have indeed been predicted, such as universal finite-size effects [5, 6, 0, 8, 9, 10, 11, 12 and amplitude relations [13. The only known example of an integrable crossover from the model $M_{p}$ to another critical point is generated by the perturbation

$$
\mathcal{L}=\mathcal{L}_{p}^{\star}+t_{p} \phi_{p(1,3)}
$$

where $\phi_{p(1,3)}$ is the weakest relevant scaling field, i.e. the field with the smallest positive RG eigenvalue, and the (dimensionful) coupling constant $t_{p}$ is positive. This crossover changes the order of criticality by one to $M_{p-1}$; this has been shown perturbatively for large values of $p$ [14, 15], by supersymmetry arguments for the crossover from the tricritical to the critical Ising model (the case $p=4$ ) [16], and more recently by thermodynamic Bethe ansatz methods for general values of $p$ [17]. 
Hence the manifold $\mathcal{C}_{p}$ of $(p-1)$-criticality is nested into all manifolds of lower criticality, as one would exspect from a mean-field analysis of the Landau-Ginzburg picture: $\mathcal{C}_{p} \subset \mathcal{C}_{p-1} \subset \ldots \subset \mathcal{C}_{3}$. In contrast to mean-field arguments, however, crossovers changing the order of criticality by more than one are induced by finetuned linear combinations of all scaling fields that are even under spin reversal, and it is not clear if any of the interpolating field theories are integrable.

The manifold of integrability also contains the leading irrelevant scaling perturbation of the critical theories $M_{p}$, 円

$$
\mathcal{L}=\mathcal{L}_{p}^{\star}-\bar{t}_{p} \phi_{p(3,1)}
$$

at least to first order in perturbation theory 4,9 . While generically a linear combination of two integrable perturbations does not generate an integrable field theory off criticality ? , the perturbations (1.1) and (1.2) share infinitely many integrals of motion so that even an arbitrary linear combination

$$
\mathcal{L}=\mathcal{L}_{p}^{\star}+t_{p} \phi_{p(1,3)}-\bar{t}_{p} \phi_{p(3,1)}
$$

should still be integrable [9]. As will be argued below, this fact is connected to the existence of nontrivial integrable lattice models in two dimensions. The presence of nonzero irrelevant coupling constants in lattice models can drastically alter their crossover behavior: since the $(p-1)$-critical lattice model is characterized by a point on $\mathcal{C}_{p}$ different from the fixed point $M_{p}$, the variation of a thermodynamic parameter causing the continuum theory $M_{p}$ to cross over to $\mathcal{C}_{p^{\prime}}\left(p^{\prime}<p\right)$ need not be tangent to $\mathcal{C}_{p^{\prime}}$ at that point, which leaves the perturbed lattice model on a less critical manifold $\mathcal{C}_{p^{\prime \prime}}\left(p^{\prime \prime}<p^{\prime}\right)$ or in a massive phase.

\footnotetext{
${ }^{1}$ The bar does not denote complex conjugation.

${ }^{2}$ The two-dimensional Ising model was studied recently [18] in the entire scaling region $\mathcal{L}=$ $\mathcal{L}^{\star}+h \phi_{(1,2)}+t \phi_{(1,3)}$, which is spanned by two integrable perturbations. The finite-size spectrum of the transfer matrix obtained by the conformal truncation method [10, 11, 12] did not show any sign of integrability except for $h=0$ or $t=0$.
} 
This paper studies such crossover phenomena by analyzing the renormalization group flow in the neighborhood of a minimal model $M_{p}$ for $p \gg 1$, where a subset of the scaling fields (including $\phi_{p(1,3)}$ and $\left.\phi_{p(3,1)}\right)$ become nearly marginal. A perturbation of $M_{p}$ can then be described by the Lagrangian

$$
\mathcal{L}=\mathcal{L}_{p}^{\star}+\sum_{i} U_{p}^{i} \Phi_{p i}
$$

the running coupling constants $U_{p}^{i}$ and their conjugate fields $\Phi_{p i}$ are defined by an expansion in the parameter $\varepsilon=4 /(p+1)$ (which is the RG eigenvalue of $\phi_{p(1,3)}$ ) [14, 15]. The $\varepsilon$-expansion can be trusted in a neighborhood $U_{p}^{i}=O(\varepsilon)$ of $M_{p}$, which contains infinitely many other fixed points $M_{p^{\prime}}$. This is an important difference to the usual $\varepsilon$-expansion about the upper critical dimension, where only two fixed points are at a distance of $O(\varepsilon)$. But at least on the trajectory linking $M_{p}$ and $M_{p-1}$, this $\varepsilon$-expansion has been shown to be a consistent RG scheme in minimal subtraction to $O\left(\varepsilon^{2}\right)[19]$.

To leading order in perturbation theory, we find in particular a unique oneparameter family of hopping trajectories $\check{U}_{p}^{i}\left(\theta, \theta_{0}\right)$ (where $\theta$ is the $\mathrm{RG}$ "time" varying along each trajectory and $\theta_{0}$ labels the trajectories). They come close to each fixed point $M_{p}$ and are self-similar in the following sense:

$$
\check{U}_{p-1}^{i}\left(\theta+\theta_{0}, \theta_{0}\right)=\check{U}_{p}^{i}\left(\theta, \theta_{0}\right)
$$

We identify these trajectories with the one-parameter family of integrable trajectories that Al.B. Zamolodchikov recently found by solving the thermodynamic Bethe ansatz for a simple factorizable scattering theory containing a single type of massive particles [四]. The flow of the $C$-function $C\left(\theta, \theta_{0}\right)$ along these trajectories is computed and seen to follow the characteristic staircase pattern that interpolates between the central charges $c_{p}$. A similar pattern is found for the flow of the anomalous dimensions $x^{(i)}\left(\theta, \theta_{0}\right)$. We shall argue that the one-parameter family $M\left(\theta_{0}\right)$ of integrable field theories defined by these $S$-matrices is described by a Lagrangian 
of the form (1.3) where both coupling constants $t_{p}$ and $\bar{t}_{p}$ are positive.

The same family of field theories can be considered for negative values of $\bar{t}_{p}$, where the RG trajectories behave very differently: they come close to only two neighboring fixed points $M_{p}$ and $M_{p-1}$ and should describe an integrable system in a $(p-2)$-phase coexistence region. This is very likely to be the eight-vertex solidon-solid model of Andrews, Baxter and Forrester (ABF) [20 in the scaling region of the so-called regime IV. The RG analysis thus establishes an intimate connection between this model and Zamolodchikov's system.

As a function of the relevant temperature-like parameter $t_{p}$, the system undergoes a second order phase transition with a rather intricate critical behavior. For $t_{p}<0$ and any value of $\bar{t}_{p}$, it is governed by the single fixed point $M_{p}$. For $t_{p}>0$ and $\bar{t}_{p}<0$, two neighboring fixed points determine the exponents; the RG confirms the scaling ansatz proposed by Huse [21] to explain the exponents in regime IV of the ABF-model. For $t_{p}>0$ and $\bar{t}_{p}>0$, they are determined by all fixed points $M_{p}, M_{p-1}, M_{p-2}, \ldots, M_{3}$ visited by the hopping trajectories.

This paper is organized as follows. In sect. 2, we write down the RG equations and determine some useful symmetry properties. Sect. 3 discusses the hopping trajectories. Sect. 4 describes the various phase coexistence regions and the critical behavior as a function of $t_{p}$. Sect. 5 contains a discussion of the results.

\section{First-order renormalization about a minimal model $M_{p}$}

To leading order in perturbation theory, the RG equations about the fixed point $M_{p}$ can be written in the form [14, 22] ㅁ

$$
\frac{\mathrm{d}}{\mathrm{d} \theta} U^{i}=y_{j}^{i} U^{j}-\pi C^{i}{ }_{j k} U^{j} U^{k}
$$

\footnotetext{
${ }^{3}$ From now on, the index $p$ will be suppressed where no ambiguities can arise.
} 
where $x^{(i)}=\left(2-y^{(i)}\right)$ are the anomalous dimensions and $C^{i}{ }_{j k}$ the structure constants of the scaling operators $\phi_{i}=\Phi_{i}(U=0)$, and $y_{j}^{i}=y^{(i)} \delta_{j}^{i}$. In Eq. (2.1), the indices run over all scaling fields that transform as scalars under rotations, including pure derivative fields $\partial_{z} \partial_{\bar{z}} \phi_{i}$. Hence the RG acts on a space of coupling constants whose dimensionality exceeds that of the thermodynamic space of the system. At any point $U$, there are linear combinations of the fields $\Phi_{i}(U)$ that are proportional to the pure derivative fields $\partial_{z} \partial_{\bar{z}} \Phi_{i}(u)$ and hence not conjugate to any thermodynamic parameter; these fields generate redundant [23] directions in coupling constant space.

The RG equations can be restricted to the "thermal" couplings that preserve the $\mathbb{Z}_{2}$-symmetry of $M_{p}$ under spin reversal. Further simplifications arise in an expansion in the parameter $\varepsilon \equiv y^{(1,3)}=4 /(p+1)$. Such an expansion is possible since both the structure constants and the scaling dimensions are analytic in $\varepsilon$. For $\varepsilon \rightarrow 0$, the scaling fields $\phi_{i}$ in the lower left corner of the Kac table (shown in fig. 1) are spectrally separated:

(i) the primary fields $\phi_{(m, n)}$ with $|m-n| \leq 1$ have dimension $x^{(m, n)} \leq 1 / 2+O(\varepsilon)$, (ii) the primary fields $\phi_{(n, n \pm 2)}$ and the (conveniently normalized [14]) descendant fields $\tilde{\phi}_{(n, n)} \equiv\left(x^{(n, n)}\right)^{-1} \partial_{z} \partial_{\bar{z}} \phi_{(n, n)}$ have dimension $2 \mp O(\varepsilon)$ and $2-O\left(\varepsilon^{2}\right)$, respectively, and

(iii) all other fields have dimension $\geq 5 / 2+O(\varepsilon)$ '

Hence the couplings $U^{(n, n \pm 2)}$ and $\tilde{U}^{(n, n)}$ become marginal in this limit, while all other couplings remain strictly relevant or irrelevant. To leading order in $\varepsilon$, the system of equations (2.1) can be truncated consistently to the nearly marginal couplings $U^{i}=O(\varepsilon)$, the other couplings remain of $O\left(\varepsilon^{2}\right)$. A convenient rescaling $U^{i}(\theta)=\varepsilon u^{i}(\varepsilon \tau) /\left(\pi C_{(1,3)(1,3)}^{(1,3)}\right)$ then brings the RG equations into the form

$$
\frac{\mathrm{d}}{\mathrm{d} \tau} u^{i}=\gamma_{j}^{i} u^{j}-c^{i}{ }_{j k} u^{j} u^{k}+O(\varepsilon)
$$

\footnotetext{
${ }^{4}$ The spectral separation breaks down for $n=O(p)$, but for the solutions of the RG equations to be discussed in the sequel, the couplings of these fields are exponentially suppressed.
} 
with $\gamma_{j}^{i}=\lim _{\varepsilon \rightarrow 0}\left(y^{i}{ }_{j} / \varepsilon\right)$ and $c^{i}{ }_{j k}=\lim _{\varepsilon \rightarrow 0}\left(C^{i}{ }_{j k} / C_{(1,3)(1,3)}^{(1,3)}\right)$.

These equations determine in particular the renormalizable manifold $\mathcal{R}_{p}$ of $M_{p}$, i.e. the set of all trajectories

$$
u_{p}^{i}(\tau) \quad \text { with } \quad u_{p}^{i}(\tau) \rightarrow 0 \text { for } \tau \rightarrow-\infty
$$

and the critical manifold $\mathcal{C}_{p}$ of $M_{p}$, i.e. the set of all trajectories

$$
u_{p}^{i}(\tau) \quad \text { with } \quad u_{p}^{i}(\tau) \rightarrow 0 \text { for } \tau \rightarrow+\infty
$$

modulo the redundant couplings (see fig. 2). The $\left(p-p^{\prime}\right)$-dimensional crossover manifold

$$
M_{p, p^{\prime}}=\mathcal{R}_{p} \cap \mathcal{C}_{p^{\prime}}
$$

describes the $\left(p-p^{\prime}-1\right)$-parameter family of field theories whose ultraviolet asymptotics is determined by $M_{p}$ and whose infrared behavior is determined by $M_{p^{\prime}}$. The simplest such solution is the unique trajectory $M_{p, p-1}$ [14],

$$
u^{(1,3)}(\tau)=\frac{\exp \left(\tau-\tau_{m}\right)}{1+\exp \left(\tau-\tau_{m}\right)}, \quad u^{i}(\tau)=0 \text { for } i \neq(1,3)
$$

where $\tau_{m}$ is a free parameter. This trajectory interpolates between $M_{p}$ and the infrared fixed point $u_{\star}^{(1,3)}=1$ associated to $M_{p-1}$.

Under a simultaneous RG time reversal and basis change involving a reflection about the diagonal of the Kac table,

$$
\tau \rightarrow-\tau, \quad \phi_{(m, n)}=\phi_{i} \rightarrow-\phi_{\bar{i}}=-\phi_{(n, m)}
$$

the equations (2.2) remain invariant since $\gamma_{\bar{j}}^{\bar{i}}=-\gamma_{j}^{i}$ and $c_{\bar{j} \bar{k}}^{\bar{i}}=c_{j k}^{i}$. Hence to every RG trajectory $u^{i}(\tau)$, there is a conjugate trajectory $\bar{u}^{i}(\tau)=-u^{\bar{i}}(-\tau)$, and to every fixed point $u_{\star}^{i}$, there is a conjugate fixed point $\bar{u}_{\star}^{i}=-u_{\star}^{\bar{i}}$. For example, the trajectory conjugate to $M_{p, p-1}$ interpolates between $M_{p}$ and the ultraviolet fixed point $u_{\star}^{(3,1)}=-1$ associated to $M_{p+1}$. Of particular importance in the sequel will be the self-conjugate trajectories, which satisfy

$$
u^{i}(\tau)=-u^{\bar{i}}\left(-\tau+\tau_{1}\right)
$$


for some value of $\tau_{1}$.

In a small neighborhood of the trajectory $M_{p, p-1}$, the RG equations can be linearized in the other couplings $v^{i} \equiv u^{i} \ll 1(i \neq(1,3))$. The equation for $u \equiv u^{(1,3)}$ then decouples and $u(\tau)$ is given by Eq. (2.6); the equations for $v^{i}$ take the form

$$
\frac{\mathrm{d}}{\mathrm{d} \tau} v^{i}=\gamma_{j}^{i}(u(\tau)) v^{j}
$$

where $\gamma_{j}^{i}(u)$ factorizes into $(3 \times 3)$-matrices

$$
\gamma^{(n)}=\left(\begin{array}{ccc}
\frac{n+1}{2} & 0 & 0 \\
0 & 0 & 0 \\
0 & 0 & -\frac{n-1}{2}
\end{array}\right)-2 u\left(\begin{array}{ccc}
\frac{n+3}{n+1} & \frac{n-1}{n+1}\left(\frac{n+2}{n}\right)^{1 / 2} & 0 \\
\frac{n-1}{n+1}\left(\frac{n+2}{n}\right)^{1 / 2} & \frac{4}{n^{2}-1} & \frac{n+1}{n-1}\left(\frac{n-2}{n}\right)^{1 / 2} \\
0 & \frac{n+1}{n-1}\left(\frac{n-2}{n}\right)^{1 / 2} & \frac{n-3}{n-1}
\end{array}\right)
$$

acting on the triplets of couplings

$$
v^{(n)}=\left(\begin{array}{c}
u^{(n, n+2)} \\
\tilde{u}^{(n, n)} \\
u^{(n, n-2)}
\end{array}\right)
$$

with $n=3,5,7, \ldots$.

A basis of solutions of Eq. (2.9) is given by the trajectories with the definite RG time reversal symmetry

$$
\left(u_{p}\left(\tau_{m}-\tau\right), v_{p}\left(\tau_{m}-\tau\right)\right)=\left(\bar{u}_{p-1}\left(\tau_{m}+\tau\right), \pm \bar{v}_{p-1}\left(\tau_{m}+\tau\right)\right)
$$

where $u_{p}(\tau)$ is given by Eq. (2.6). For each value of $n$, there is precisely one linearly independent even solution $v_{p}^{(n)+}$ and two linearly independent odd solutions $v_{p}^{(n)-}$ and $v_{p}^{(n) 0}$. The couplings $v_{p}^{(n) 0}$ are conjugate to the pure derivative fields $\partial_{z} \partial_{\bar{z}} \Phi_{(n, n)}(u)$ of of $M_{p, p-1}$ and play a redundant rôle.

For the asymptotic behavior of the trajectories as $\tau \rightarrow-\infty$ (i.e. $u_{p} \equiv u \rightarrow 0$ ), there are three possibilities. For every value of $n$, there is one linearly independent 
solution of (2.9),

$$
v_{p}^{(n) r}(u)=u^{\frac{n+1}{2}}\left[\left(\begin{array}{l}
1 \\
0 \\
0
\end{array}\right)+u\left(\begin{array}{c}
-\frac{2(n+3)}{n+1} \\
-\frac{4(n-1)}{(n+3)(n+1)}\left(\frac{n+2}{n}\right)^{1 / 2} \\
0
\end{array}\right)+O\left(u^{2}\right)\right],
$$

defining a renormalizable trajectory $\left(u_{p}(\tau), v_{p}^{(n) r}(u(\tau))\right)$ and one solution

$$
v_{p}^{(n) 0}(u)=\left(\begin{array}{c}
0 \\
1 \\
0
\end{array}\right)+u\left(\begin{array}{c}
\frac{4}{n+1}\left(\frac{n+2}{n}\right)^{1 / 2} \\
-\frac{8}{n^{2}-1} \\
-\frac{4}{n-1}\left(\frac{n-2}{n}\right)^{1 / 2}
\end{array}\right)+O\left(u^{2}\right)
$$

defining a redundant trajectory. Any solution that is linearly independent from (2.13) and (2.14) describes a theory that is nonrenormalizable about $M_{p}$. Conversely, there is one linearly independent solution $v_{p}^{(n) c}(u)$ that defines a trajectory in $\mathcal{C}_{p-1}$; any solution that is linearly independent of $v_{p}^{(n) c}(u)$ and $v_{p}^{(n) 0}(u)$ is of lower criticality.

\section{Self-similar hopping trajectories}

In this section, we study the RG flow of a self-conjugate perturbation of the fixed point $M_{p}$,

$$
u_{p}^{i}(\tau=0)=\bar{u}_{p}^{i}(\tau=0) \ll 1,
$$

corresponding to a point in theory space that is much closer to $M_{p}$ than any of the other fixed points $M_{p^{\prime}}$. We define the parameter

$$
s \equiv u_{p}^{(1,3)} \bar{u}_{p}^{(1,3)}>0
$$

It is easy to verify that there is a unique one-parameter family $\hat{u}_{p}^{i}(\tau, s)$ of trajectories that satisfy the conditions (3.1) and $\hat{u}_{p}^{i}(\tau, s) \subset \mathcal{C}_{p-1}$, i.e. $\hat{v}_{p}$ can be expanded 
in the basis of triplets $v_{p}^{(n) c}$ and $v_{p}^{(n) 0}$. f Self-conjugacy then dictates $\hat{u}_{p}^{i}(\tau, s) \subset \mathcal{R}_{p+1}$ as well and therefore

$$
\hat{u}_{p}^{i}(\tau, s) \subset M_{p+1, p-1}
$$

Any trajectory in $M_{p+1, p-1}$ with a given value of $s$ differs from $\hat{u}_{p}^{i}(\tau, s)$ only by spurious couplings $v_{p}^{(n) 0}$, hence these trajectories (shown in fig. 3a) span the twodimensional crossover manifold $M_{p+1, p-1}$.

Consider now the one-parameter family $\check{u}_{p}^{i}(\tau, s)$ of trajectories that satisfy the condition (3.1) and are even in the sense of Eq. (2.12) up to spurious couplings, i.e. $v_{p}$ can be expanded in the basis of triplets $v_{p}^{(n)+}$ and $v_{p}^{(n) 0}$. The trajectory $\check{u}_{p}^{i}(\tau, s)$ is self-similar (see fig. 3b) after a scaled RG time $\tau_{0}=\varepsilon \theta_{0} \simeq \log (1 / s)$ for small $s$ :

$$
\check{u}_{p-1}^{i}\left(\tau_{0}, s\right)=\overline{\check{u}}_{p}^{i}(0, s)=\check{u}_{p}^{i}(0, s)
$$

It comes close to each fixed point $M_{p^{\prime}}$ in the time interval

$$
\left(p-p^{\prime}-\frac{1}{2}\right) \theta_{0} \lesssim \theta \lesssim\left(p-p^{\prime}+\frac{1}{2}\right) \theta_{0}
$$

and up to a minimum distance given by the parameter $s_{p^{\prime}}=s(1+O(\varepsilon))$, whereafter it hops to the next lower fixed point. It is again easy to check that up to spurious couplings, this is the only self-similar trajectory for that value of $s$. Thus the

\footnotetext{
${ }^{5}$ For a given value of $s$, one has $\hat{u}_{p}^{(1,3)}=s^{1 / 2}=-\hat{u}_{p}^{(3,1)}$ by Eqns. (3.1) and (3.2). The remaining
} couplings are recursively determined by the equations

$$
\hat{v}_{p}^{(n)}(0, s)=a_{(n) c}(s) v_{p}^{(n) c}\left(u=s^{1 / 2}\right)+a_{(n) 0}(s) v_{p}^{(n) 0}\left(u=s^{1 / 2}\right)
$$

and the self-conjugacy conditions

$$
\hat{u}_{p}^{(n, n+2)}(0, s)=-\hat{u}_{p}^{(n+2, n)}(0, s) \quad \text { and } \quad \hat{\tilde{u}}_{p}^{(n, n)}(0, s)=0
$$

Analogous recursion relations hold for the trajectories $\check{u}_{p}^{i}(\tau, s)$ below. Notice that for both families and every value of $n$, the ratio of the relevant and the irrelevant coupling $u_{p}^{(n, n+2)}(0, s) / u_{p}^{(n, n-2)}(0, s)$ in the $n$th triplet goes to 0 as $s \rightarrow 0$, hence the couplings with higher $n$ are strongly suppressed for small $s$ and $\tau$. 
trajectories $\check{u}_{p}^{i}\left(\tau, s=s\left(\theta_{0}\right)\right)$ define a unique one-parameter family of field theories $M\left(\theta_{0}\right)$

The RG flow of the $C$-function 14

$$
C\left(u_{p}^{i}\right)=c_{p}+\frac{3 \varepsilon^{3}}{16}\left(-3 \gamma_{i j} u_{p}^{i} u_{p}^{j}+2 c_{i j l} u_{p}^{i} u_{p}^{j} u_{p}^{l}\right)+O\left(\varepsilon^{4}\right)
$$

for the theory $M\left(\theta_{0}\right)$ satisfies

$$
C\left(\theta, \theta_{0}\right)-c_{p}=C\left(\theta+\theta_{0}, \theta_{0}\right)-c_{p-1}+O\left(\varepsilon^{4}\right)
$$

and in particular for integer $k$

$$
\begin{gathered}
C\left(k \theta_{0}, \theta_{0}\right)=c_{p-k}+O\left(\varepsilon^{4}\right) \\
C\left(\left(k+\frac{1}{2}\right) \theta_{0}, \theta_{0}\right)=c_{p-k}-\frac{1}{2}\left(c_{p-k}-c_{p-k-1}\right)+O\left(\varepsilon^{4}\right)
\end{gathered}
$$

by Eq. (3.4). A step of this self-repeating staircase pattern for several values of $\tau_{0}$ is shown in fig. 4, which was obtained by numerical integration of Eq. (2.9). [

The anomalous dimensions $x^{(i)}$, i.e. the eigenvalues of the matrix

$$
2 \delta^{i}{ }_{j}-y_{j}^{i}\left(u_{p}^{l}\right)=2 \delta^{i}{ }_{j}-y_{j(p)}^{i}+2 \varepsilon c_{j l}^{i} u_{p}^{l}+O\left(\varepsilon^{2}\right)
$$

show a very similar pattern. For example, the spectral flow associated to the second subdiagonals of the Kac table satisfies

$$
\begin{aligned}
& x^{(n, n+2)}\left(\theta+\theta_{0}, \theta_{0}\right)=x^{(n-2, n)}\left(\theta, \theta_{0}\right)+O\left(\varepsilon^{2}\right) \\
& x^{(n, n-2)}\left(\theta+\theta_{0}, \theta_{0}\right)=x^{(n+2, n)}\left(\theta, \theta_{0}\right)+O\left(\varepsilon^{2}\right)
\end{aligned}
$$

and in particular for integer $k$

$$
\begin{array}{ll}
x^{(n, n+2)}\left(k \theta_{0}, \theta_{0}\right)=x_{p}^{(n-2 k, n+2-2 k)}+O\left(\varepsilon^{2}\right) & (2 k<n) \\
x^{(n, n-2)}\left(k \theta_{0}, \theta_{0}\right)=x_{p}^{(n+2 k, n-2+2 k)}+O\left(\varepsilon^{2}\right) & (2 k>-n),
\end{array}
$$

\footnotetext{
${ }^{6}$ Eqns. (3.3) and (3.4) also indicate the possibility that there exists a two-parameter family of trajectories $u_{p}^{i}(\tau, \hat{s}, \check{s})$ with $u_{p}^{i}(\tau, \hat{s}, \check{s}) \rightarrow \breve{u}_{p}^{i}(\tau, \check{s})$ as $\hat{s} \rightarrow 0$ and $u_{p}^{i}(\tau, \hat{s}, \check{s}) \rightarrow \hat{u}_{p}^{i}(\tau, \hat{s})$ as $\breve{s} \rightarrow 0$, their $C$-function being a staircase pattern where all steps have approximately the same length except the step at $c_{p}$, which is shorter.
} 
and similar equations hold for the other fields.

Thus the field theories $M\left(\theta_{0}\right)$ behave under RG transformations in a strikingly similar way to the one-parameter family of integrable systems with a single type of massive particles characterized by the factorizable $S$-matrix [四]

$$
S\left(\rho, \theta_{0}\right)=\frac{\sinh \rho-i \cosh 2 \theta_{0}}{\sinh \rho+i \cosh 2 \theta_{0}}
$$

written in terms of the Lorentz-invariant rapidity difference $\rho$. Since the selfsimilarity (3.4) is unique to the the theories $M\left(\theta_{0}\right)$, we are lead to identify them with this type of integrable system. It is plausible that the hopping trajectories $\check{u}_{p}^{i}(\tau, s)$ describe integrable systems since the bare Lagrangian $\left.(\partial / \partial s) \sum_{i}\left(\check{U}_{p}^{i} \Phi_{p i}\right)(0, s)\right|_{s=0}$ is of the form (1.3) with $t_{p}>0$ and $\bar{t}_{p}>0$, but it is difficult to make such a statement precise within the $\varepsilon$-expansion since these trajectories are nonrenormalizable about any minimal model $M_{p^{\prime}}$.

The following scaling argument indicates, however, that the Lagrangian

$$
\mathcal{L}_{p}=\mathcal{L}_{p}^{\star}+\sum_{i} t_{p}^{i} \phi_{p i}
$$

for the theories $M\left(\theta_{0}\right)$ is precisely (1.3) for any value of $p$. We define the dimensionless scaling variables

$$
s_{p}^{i} \equiv t_{p}^{i} t_{p}^{\omega_{p}^{i}}, \quad \bar{s}_{p}^{i} \equiv t_{p}^{i} \bar{t}_{p}^{i}
$$

where $\omega_{p}^{i}$ and $\bar{\omega}_{p}^{i}$ are the crossover exponents

$$
\omega_{p}^{i} \equiv-\frac{y_{p}^{(i)}}{y_{p}}, \quad \bar{\omega}_{p}^{i} \equiv-\frac{y_{p}^{(i)}}{\bar{y}_{p}},
$$

with

$$
y_{p} \equiv y_{p}^{(1,3)}=\frac{4}{p+1}, \quad \bar{y}_{p} \equiv y_{p}^{(3,1)}=-\frac{4}{p} .
$$

The theory $M_{p, p-1}$ has $s_{p}^{i}=0$ for $i \neq(1,3)$ and $\bar{s}_{p-1}^{i}=0$ for $i \neq(3,1)$, while for any finite value of $\theta_{0}$, the theory $M\left(\theta_{0}\right)$ must have irrelevant couplings

$$
s_{p}^{i} \ll 1 \quad \text { for } \theta_{0} \gg 1
$$


since its ultraviolet behavior differs from $M_{p}$, and relevant couplings

$$
s_{p-1}^{i} \ll 1 \quad \text { for } \theta_{0} \gg 1
$$

since its infrared behavior differs from $M_{p-1}$. There is an analytic mapping between the two sets of couplings (3.17) and (3.18), which is just a coordinate transformation on theory space [19]. The most relevant coupling $s_{p-1}^{\mathrm{rel}}$ of the set $(3.18)$ and the most irrelevant coupling $s_{p}^{\mathrm{irr}} \sim s_{p-1}^{\mathrm{rel}}$ of the set 3.17 determine the logarithmic scale intervals (the RG time intervals) in which the theory $M\left(\theta_{0}\right)$ is governed by the fixed points $M_{p}$ and $M_{p-1}$, respectively:

$$
\exp \left(\Delta \theta_{p}\right) \sim\left(s_{p}^{\mathrm{irr}}\right)^{1 / y_{p}^{\mathrm{irr}}} \gg 1, \quad \exp \left(\Delta \theta_{p-1}\right) \sim\left(s_{p-1}^{\mathrm{rel}}\right)^{-1 / y_{p-1}^{\mathrm{rel}}} \gg 1
$$

Zamolodchikov's solution [1] of the thermodynamic Bethe ansatz equations for the $S$-matrix (3.12) says that Eq. (3.5) is valid beyond perturbation theory, i.e. $\Delta \theta_{p}=$ $\Delta \theta_{p-1}=\theta_{0}$. This dictates

$$
\frac{1}{y_{p}^{\mathrm{irr}}}=-\frac{1}{y_{p-1}^{\mathrm{rel}}},
$$

which can be satisfied only if $t_{p}^{\mathrm{irr}} \sim t_{p}^{(3,1)}$ and $t_{p-1}^{\mathrm{rel}} \sim t_{p-1}^{(1,3)}$, and hence $y_{p}^{\mathrm{irr}}=-4 / p=$ $-y_{p-1}^{\text {rel }}$ by Eq. (3.16). Repeating the argument for $p^{\prime}=p+1$ then fixes the form of the Lagrangian (1.3). Hence from the Lagrangian point of view, the scale hopping of the theories $M\left(\theta_{0}\right)$ is caused by an intricate interplay of the relevant field $\phi_{(1,3)}$ and the irrelevant field $\phi_{(3,1)}$ under the renormalization group.

The scaling parameter $s_{p} \equiv \bar{t}_{p} t_{p}^{\omega_{p}}\left(\right.$ with $\left.\omega_{p} \equiv \omega_{p}^{(3,1)}=(p+1) / p\right)$ can be expressed by

$$
s_{p}=g_{p}^{\omega_{p}} \bar{g}_{p} \exp \left(\bar{y}_{p} \theta_{0}\right)
$$

in terms of $\theta_{0}$ and the dimensionless coupling constants

$$
g_{p}=t_{p} \xi_{p, p-1}^{y_{p}}, \quad \bar{g}_{p}=\bar{t}_{p} \xi_{p+1, p}^{\bar{y}_{p}}
$$

Here $\xi_{p, p-1}$ and $\xi_{p+1, p}$ denote the crossover length scales of $M_{p, p-1}$ and $M_{p+1, p}$, respectively. Unlike the running couplings, $g_{p}$ and $\bar{g}_{p}$ are measurable parameters related 
to universal amplitude relations [13]. By comparing the solution of the thermodynamic Bethe ansatz with conformal perturbation theory, they can be computed to arbitrarily high accuracy [6, 17]. To leading order in the $\varepsilon$-expansion, one obtains $\square$

$$
g_{p}=\bar{g}_{p}=\frac{\varepsilon}{\pi C_{(1,3)(1,3)}^{(1,3)}}+O\left(\varepsilon^{2}\right) .
$$

\section{Phase coexistence and critical behavior}

The integrability of the theories given by the Lagrangian (1.3) should not depend on the sign of the two coupling constants $t_{p}$ and $\bar{t}_{p}$. However, the behavior of the $\mathrm{RG}$ trajectories and hence the long-distance structure crucially depends on these signs: the four one-parameter families of field theories

$$
M_{p}^{++}\left(s_{p}\right)=M\left(\theta_{0}\left(s_{p}\right)\right), \quad M_{p}^{+-}\left(s_{p}\right), \quad M_{p}^{-+}\left(s_{p}\right), \quad M_{p}^{--}\left(s_{p}\right)
$$

labeled by the scaling parameter $s_{p}$ and the signs of $t_{p}$ and $\bar{t}_{p}$, describe the system in different thermodynamic phases which we discuss below. The qualitative RG scenario and the resulting phase diagram in the $\left(t_{p}, \bar{t}_{p}\right)$-plane are shown in fig. 5. As a function of the relevant parameter $t_{p}$, the system undergoes a second order phase transition whose exponents depend on the phase.

\subsection{The theories $M_{p}^{++}$}

For $t_{p}>0$ and $\bar{t}_{p}>0$, the solutions of the RG equations are the self-similar hopping trajectories $\check{u}_{p}^{i}$. Following such a trajectory down to $M_{3}$ shows that also $t_{3}>0$; the system is in a disordered high-temperature phase. As $t_{p} \rightarrow 0$, these trajectories come arbitrarily close to all fixed points $M_{p^{\prime}}$. This implies that the leading thermodynamic singularities are governed by the fixed points $M_{p^{\prime}}$ with $p^{\prime} \leq p$, while the other fixed points contribute corrections to scaling. Exact critical exponents for these theories will be reported in a forthcoming publication [24].

\footnotetext{
${ }^{7}$ Hence to this order, $s_{p}$ coincides with the parameter $s$ defined in Eq. (3.2).
} 


\subsection{The theories $M_{p}^{+-}$}

The trajectories for $t_{p}>0$ and $\bar{t}_{p}<0$ are obtained by analytically continuing the solutions $\check{u}_{p}^{i}=\left(\check{u}_{p}, \check{v}_{p}\right)$ of regime 1 to $\left(\check{u}_{p},-\check{v}_{p}\right)$ in the neighborhood of $M_{p, p-1}$, i.e. at times $0 \lesssim \theta \lesssim \theta_{0}(s)$; these solutions are still even under RG time reversal according to Eq. (2.12) up to spurious couplings. At times $\theta \gtrsim \theta_{0}(s)$, they approach the trajectory

$M_{p-1}^{(-)}$generated by the integrable perturbation (1.1) of $M_{p-1}$ with $t_{p-1}<0$ which describes the system in a low-temperature region of $p-2$ coexisting phases. It is likely that this one-parameter family of solutions shares the same infrared behavior. Hence they are very different from the self-similar trajectories: they come close to only two fixed points $M_{p}$ and $M_{p-1}$ and run away in both time limits,

$$
\bar{u}_{p}(\theta, s) \rightarrow-\infty \text { as } \theta \rightarrow-\infty, \quad u_{p-1}(\theta, s) \rightarrow-\infty \text { as } \theta \rightarrow+\infty
$$

An ordered phase above the critical "temperature" $t_{p}=0$ is not to be expected for the continuum theory, but it does occur in regime IV of the ABF lattice model. It is easy to show that the above renormalization group picture indeed reproduces the correct order parameter exponents known from the exact solution. The behavior of the trajectories indicates that the theories $M_{p}^{+-}\left(s_{p}\right)$ are characterized by two length scales, the crossover scale $\xi_{p, p-1}$ and the inverse mass $\xi$. Their asymptotic temperature dependence is given by

$$
\xi_{p, p-1} \sim t_{p}^{-1 / y_{p}}
$$

and

$$
\frac{\xi}{\xi_{p, p-1}} \sim \exp \theta_{0} \sim t_{p}^{\omega_{p} / \bar{y}_{p}}=t_{p}^{-\omega_{p} / y_{p-1}},
$$

as follows from Eqns. (3.21) and (3.16). For the leading singular behavior of the order parameters

$$
\left\langle\phi_{p(n, n)}\right\rangle \sim \xi_{p, p-1}^{-x_{p}^{(n, n)}}\left(\frac{\xi}{\xi_{p, p-1}}\right)^{-x_{p-1}^{(n, n)}}
$$

we obtain therefore

$$
\left\langle\phi_{p(n, n)}\right\rangle \sim t_{p}^{\beta_{p}^{(n, n)}+\omega_{p} \beta_{p-1}^{(n, n)}}
$$


with $\beta_{p}^{(n, n)}=x_{p}^{(n, n)} / y_{p}$, which is precisely Huse's result [21. We conclude that the theories $M_{p}^{+-}$describe the ABF model in the scaling region of regime IV. This explains the phase structure as a consequence of the same interplay of $\phi_{1,3}$ and $\phi_{3,1}$ that causes the scale hopping of the theories $M_{p}^{++}$.

\subsection{The theories $M_{p}^{-+}$}

For $t_{p}<0$ and $\bar{t}_{p}>0$, the trajectories are conjugate to those of $M_{p}^{+-}$and in fact just those of $M_{p+1}^{+-}$; they describe the system in the $(p-1)$-phase coexistence region. At times $-\theta_{0}(s) \lesssim \theta \lesssim 0$, they come close to the fixed point $M_{p+1}$, the trajectory $M_{p+1, p}$, and the fixed point $M_{p}$; at large times, they run away,

$$
\bar{u}_{p+1} \rightarrow-\infty \text { as } \theta \rightarrow-\infty, \quad u_{p} \rightarrow-\infty \text { as } \theta \rightarrow+\infty
$$

The critical behavior as $t_{p} \rightarrow 0$ is governed by the fixed point $M_{p}$, with corrections to scaling due to the irrelevant operator $\phi_{p(3,1)}$.

\subsection{The theories $M_{p}^{--}$}

For $t_{p}<0$ and $\bar{t}_{p}<0$ (hence $s>0$ ), one exspects solutions that are again selfconjugate, $u_{p}^{i}(\theta, s)=-\bar{u}_{p}^{i}(-\theta, s)$, and describe the system in the $(p-1)$-phase coexistence region. They come close only to one fixed point $M_{p}$, and run away at large times,

$$
\bar{u}_{p}^{(1,3)} \rightarrow-\infty \text { as } \theta \rightarrow-\infty, \quad u_{p}^{(1,3)} \rightarrow-\infty \text { as } \theta \rightarrow+\infty
$$

The theories $M_{p}^{--}$should describe the ABF models in the scaling region of regime III. The critical behavior is governed by $M_{p}$, but the corrections to scaling are of opposite sign compared to the theories $M_{p}^{-+}$. 


\section{Discussion}

We have studied perturbations of a minimal conformal field theory $M_{p}$ by a linear combination of the scaling fields $\phi_{(1,3)}$ and $\phi_{(3,1)}$. This generates four oneparameter families of massive integrable field theories $M_{p}^{++}\left(s_{p}\right), M_{p}^{+-}\left(s_{p}\right), M_{p}^{-+}\left(s_{p}\right)$ and $M_{p}^{--}\left(s_{p}\right)$, which are labeled by the signs of the two coupling constants and the dimensionless scaling parameter $s_{p}$, and describe the system in different phases off criticality.

The disordered high-temperature phase corresponds to the theories $M_{p}^{++}\left(s_{p}\right)$, which are related to Zamolodchikov's scattering theory (3.12), and show a novel behavior under the renormalization group: the trajectories come close to many fixed points $M_{p^{\prime}}$ for a certain RG time interval $\theta_{0}\left(s_{p}\right)$, whereafter they hop to the next fixed point $M_{p^{\prime}-1}$. The correlation functions of these theories are characterized by a multitude of crossover length scales $\xi_{p, p-1}$; any two subsequent such scales have the same ratio $\xi_{p-1, p-2} / \xi_{p, p-1}=\mathrm{e}^{\theta_{0}}$.

To leading order in an $\varepsilon$-expansion, we have shown that the RG equations have indeed a unique one-parameter family of solutions with this behavior, which is tied to the simultaneous presence of relevant and irrelevant coupling constants with scaling dimensions of $O(\varepsilon)$.

The theories $M_{p}^{+-}\left(s_{p}\right)$ and $M_{p}^{--}\left(s_{p}\right)$ are argued to describe the scaling region of the ABF lattice models in regime III and IV, respectively. It would be interesting to study the corrections to scaling in these exactly solved models. Are all nonanalytic corrections due to irrelevant operators in the family of $\phi_{(3,1)}$ ? This would severely restrict the possible lattice effects. And is it possible to find lattice models with the leading irrelevant coupling of opposite sign, that would hence be in Zamolodchikov's phase?

Note added: After the draft of this paper had been completed, I received a copy of ref. [25], where Zamolodchikov's $S$-matrix for antiperiodic boundary conditions 
is associated to the $D$ series of minimal models. The issue of boundary conditions deserves further study. The Lagrangian description suggests that integrable systems with scale hopping trajectories should exist in the $A$ and $D$ series.

\section{Acknowledgments}

I enjoyed helpful discussions with John L. Cardy, who also gave the manuscript a critical reading, and with Reinhard Lipowsky. 


\section{References}

[1] Al.B. Zamolodchikov, Resonance factorized scattering and roaming trajectories, Paris preprint ENS-LPS-335 (1991)

[2] A.A. Belavin, A.M. Polyakov, and A.B. Zamolodchikov, Nucl. Phys. B241 (1984), 333

[3] A.B. Zamolodchikov, Yad. Fiz. 44 (1986), 82 [Sov. J. Nucl. Phys. 44 (1986), $529]$

[4] A.B. Zamolodchikov, JETP Lett. 46 (1987), 160; Int. Journ. Mod. Phys. A3 (1988), 743; Adv. Stud. Pure Math. 19 (1989), 1

[5] M. Lüscher, in Progress in Gauge Field Theory (Cargèse 1983), ed. G. 't Hooft et al., Plenum, New York 1984; Comm. Math. Phys. 104 (1986), 177; in Champs, Cordes, et Phénomènes Critiques, proceedings of the 1988 Les Houches Summer School, ed. E. Brézin and J. Zinn-Justin, North Holland, Amsterdam, 1989

[6] Al.B. Zamolodchikov, Nucl. Phys. B 342 (1990), 695

[7] Al.B. Zamolodchikov, Nucl. Phys. B 348 (1991), 619

[8] T.R. Klassen, E. Melzer, Nucl.Phys. B 338 (1990), 485

[9] Al.B. Zamolodchikov, Nucl. Phys. B 358 (1991), 497

[10] V.P. Yurov, Al.B. Zamolodchikov, Intern. Journ. Mod. Phys. A5 (1990), 3221; Trieste preprint SISSA 36 EP (1990)

[11] M. Lässig, G. Mussardo and J.L. Cardy, Nucl. Phys. B348 (1991), 591

[12] M. Lässig, M. Martins, Nucl. Phys. B354 (1991), 666 
[13] M. Lässig, Exact universal amplitude ratios in two-dimensional systems near criticality, Santa Barbara preprint UCSBTH 91-20

[14] A.B. Zamolodchikov, Yad. Fiz. 46 (1987), 1819 [Sov. J. Nucl. Phys. 46 (1987), 1090]

[15] A.W.W. Ludwig, J.L. Cardy, Nucl. Phys. B285 (1987), 687

[16] D.A. Kastor, E.J. Martinec, S.H. Shenker, Nucl. Phys. B316 (1989), 590

[17] Al.B. Zamolodchikov, Nucl. Phys. B358 (1991), 524

[18] M. Lässig, M. Martins, unpublished

[19] M. Lässig, Nucl. Phys. B334 (1990), 652

[20] G.E. Andrews, R.J. Baxter, P.J. Forrester, J. Stat. Phys. 35 (1984), 193

[21] D.A. Huse, J. Phys. A 16 (1983), 4357; Phys. Rev. B 30 (1984), 3908

[22] J.L. Cardy, in Champs, Cordes et Phénomènes Critiques, proceedings of the 1988 Les Houches Summer School, ed. E. Brézin and J. Zinn-Justin, Elsevier, 1989

[23] F.J. Wegner, in Phase Transitions and Critical Phenomena, Vol. 6B, ed. C. Domb and M.S. Green, Academic Press, London, 1976

[24] M. Lässig, Exact critical exponents of the staircase model, Jülich preprint, December 1991

[25] T.R. Klassen, E. Melzer, Cornell preprint CLNS-91/1111 


\section{Figure Captions}

1. Positions of the nearly marginal thermal operators in the Kac table of a unitary minimal model $M_{p}$ for $p \gg 1$. The operators $\phi_{(n, n+2)}$ are relevant, while the operators $\phi_{(n, n-2)}$ and $\tilde{\phi}_{(n, n)}$ are irrelevant.

2. Special solutions of the $\mathrm{RG}$ equations in the vicinity of $M_{p, p-1}$ (schematic). (a) A trajectory in $\mathcal{R}_{p}$. (b) A trajectory in $\mathcal{C}_{p-1}$. (c) A redundant trajectory.

3. Self-conjugate trajectories in the vicinity of the fixed point $M_{p}$ (schematic). (a) A trajectory in $M_{p+1, p-1}$. (b) A self-similar trajectory.

4. The $C$-function $C\left(\tau, \tau_{0}\right)$ of the unique self-similar trajectory $\check{u}_{p}^{i}\left(\tau, s\left(\tau_{0}\right)\right)$ : a step in the staircase pattern for $\tau_{0}=3.2,3.6,4.0$ and 4.4 (solid lines). For larger values of $\tau_{0}$, the steps get more pronounced as the solutions tend towards the limit trajectory $M_{p, p-1}$ (long-dashed line).

5. (a) The RG flow in the vicinity of $M_{p}$. A self-similar trajectory of regime 1 (solid line) visits all fixed points, trajectories in regime 2 or 3 (long-dashed lines) visit two fixed points, and a trajectory in regime 4 (short-dashed line) visits only one fixed point. (b) The resulting phase diagram in the $\left(t_{p}, \bar{t}_{p}\right)$ plane. 